\title{
Outbreak of Plague in a High Malaria Endemic Region - Nyimba District, Zambia, March-May 2015
}

\author{
Nyambe Sinyange, MSc ${ }^{1,5}$; Ramya Kumar, MPH ${ }^{2,6}$; Akatama Inambao ${ }^{1,5}$; Loveness Moonde, $\mathrm{MPH}^{1,5}$; Jonathan Chama, $\mathrm{MBChB}^{3}$; Mapopa Banda ${ }^{3}$; \\ Elliot Tembo ${ }^{3}$; Beron Nsonga ${ }^{3}$; John Mwaba ${ }^{4}$; Sombo Fwoloshi, MBChB ${ }^{4,5}$; Kebby Musokotwane, MSc ${ }^{3}$; Elizabeth Chizema, MPH ${ }^{3}$; \\ Muzala Kapin'a, $\mathrm{MSc}^{3}$; Benard Mudenda Hang'ombe, $\mathrm{PhD}^{7}$; Henry C. Baggett, $\mathrm{MD}^{2,6}$; Lottie Hachaambwa, $\mathrm{MBChB}^{5,8}$
}

Outbreaks of plague have been recognized in Zambia since 1917 (1). On April 10, 2015, Zambia's Ministry of Health was notified by the Eastern Provincial Medical Office of possible bubonic plague cases in Nyimba District. Eleven patients with acute fever and cervical lymphadenopathy had been evaluated at two rural health centers during March 28April 9, 2015; three patients died. To confirm the outbreak and develop control measures, the Zambia Ministry of Health's Field Epidemiology Training Program (ZFETP) conducted epidemiologic and laboratory investigations in partnership with the University of Zambia's schools of Medicine and Veterinary Medicine and the provincial and district medical offices. Twenty-one patients with clinically compatible plague were identified, with symptom onset during March 26-May 5, 2015. The median age was 8 years, and all patients were from the same village. Blood specimens or lymph node aspirates from six (29\%) patients tested positive for Yersinia pestis by polymerase chain reaction (PCR). There is an urgent need to improve early identification and treatment of plague cases. PCR is a potential complementary tool for identifying plague, especially in areas with limited microbiologic capacity. Twelve (57\%) patients, including all six with PCR-positive plague and all three who died, also tested positive for malaria by rapid diagnostic test (RDT). Plague patients coinfected with malaria might be misdiagnosed as solely having malaria, and appropriate antibacterial treatment to combat plague might not be given, increasing risk for mortality. Because patients with malaria might be coinfected with other pathogens, broad spectrum antibiotic treatment to cover other pathogens is recommended for all children with severe malaria, until a bacterial infection is excluded.

\section{Epidemiologic Investigation}

After the initial report of possible plague cases, the Nyimba District Medical Office began active case finding in the health center catchment areas. During March 26-May 5, 2015, a total of 111 patients with fever or recent history of fever, and swollen lymph nodes were identified and evaluated for possible plague; $82(75 \%)$ patients were admitted to the health centers, treated with intravenous gentamycin and benzyl penicillin, and observed for at least one night. The remaining 29 patients received oral doxycycline and cotrimoxazole as outpatients.
The ZFETP investigation team arrived on April 29 to support the local response. Illness clinically compatible with plague was defined as temperature $\geq 100.4^{\circ} \mathrm{F}\left(\geq 38^{\circ} \mathrm{C}\right)$ or history of recent fever, and at least one of the following signs or symptoms: 1) painful, visibly enlarged cervical, axillary, or inguinal lymph nodes; 2) evidence of sepsis (prostration, reduced responsiveness, or hypotension); or 3) severe pneumonia (cough with respiratory distress or hemoptysis). A suspected case of plague was defined as a clinically compatible illness in a resident of Nyimba District, with symptom onset during March 15-May 5.

Among the 82 patients who had been hospitalized, 25 (not including any of the 21 identified with clinically compatible plague with symptom onset during March 26-May 5, 2015) were available for physical examination. None had lymph node enlargement consistent with buboes, and none met the case definition. Based on medical record review, among all 82 hospitalized patients, 21 (26\%) from the same village met the suspected plague case definition. Among these patients, the earliest reported symptom onset was March 26, and the number of cases peaked during April 6-7 (Figure). The median age was 8 years (range $=3-18$ years), and $95 \%$ were aged $<15$ years. Eleven $(52 \%)$ patients were male. After fever, the most common signs and symptoms were swollen cervical lymph nodes $(90 \%)$, cough $(38 \%)$, and headache $(33 \%)$ (Table 1). Two patients who died lacked documentation of enlarged nodes, although both had reported neck pain.

Among the 21 suspected cases, blood specimens were collected from $11(52 \%)$ patients, and lymph node aspirates were collected from three (14\%) patients for culture. Both blood and lymph node aspirates were obtained from two patients (Table 2). Specimens were stored at the district hospital laboratory at $39^{\circ} \mathrm{F}-46^{\circ} \mathrm{F}\left(4^{\circ} \mathrm{C}-8^{\circ} \mathrm{C}\right)$ before being transported to the University Teaching Hospital reference laboratory in Lusaka, a process that took 3-17 days. Blood from four patients was placed in ethylenediaminetetraacetic acid (EDTA)-containing tubes, making these specimens unsuitable for culture. The remaining seven blood specimens remained negative for $Y$.pestis after 7 days, including one that likely grew a contaminant. All three lymph node aspirates were culture-negative. Five blood specimens and two lymph node aspirates (six patients) tested positive for $Y$. pestis by PCR targeting a 478-base pair region of the $Y$. pestis plasminogen activator gene (2) (Table 2). 
FIGURE. Symptom onset date, outcome, and epidemiologic and laboratory confirmation status of 21 plague cases — Nyimba District, Zambia, March-May, 2015

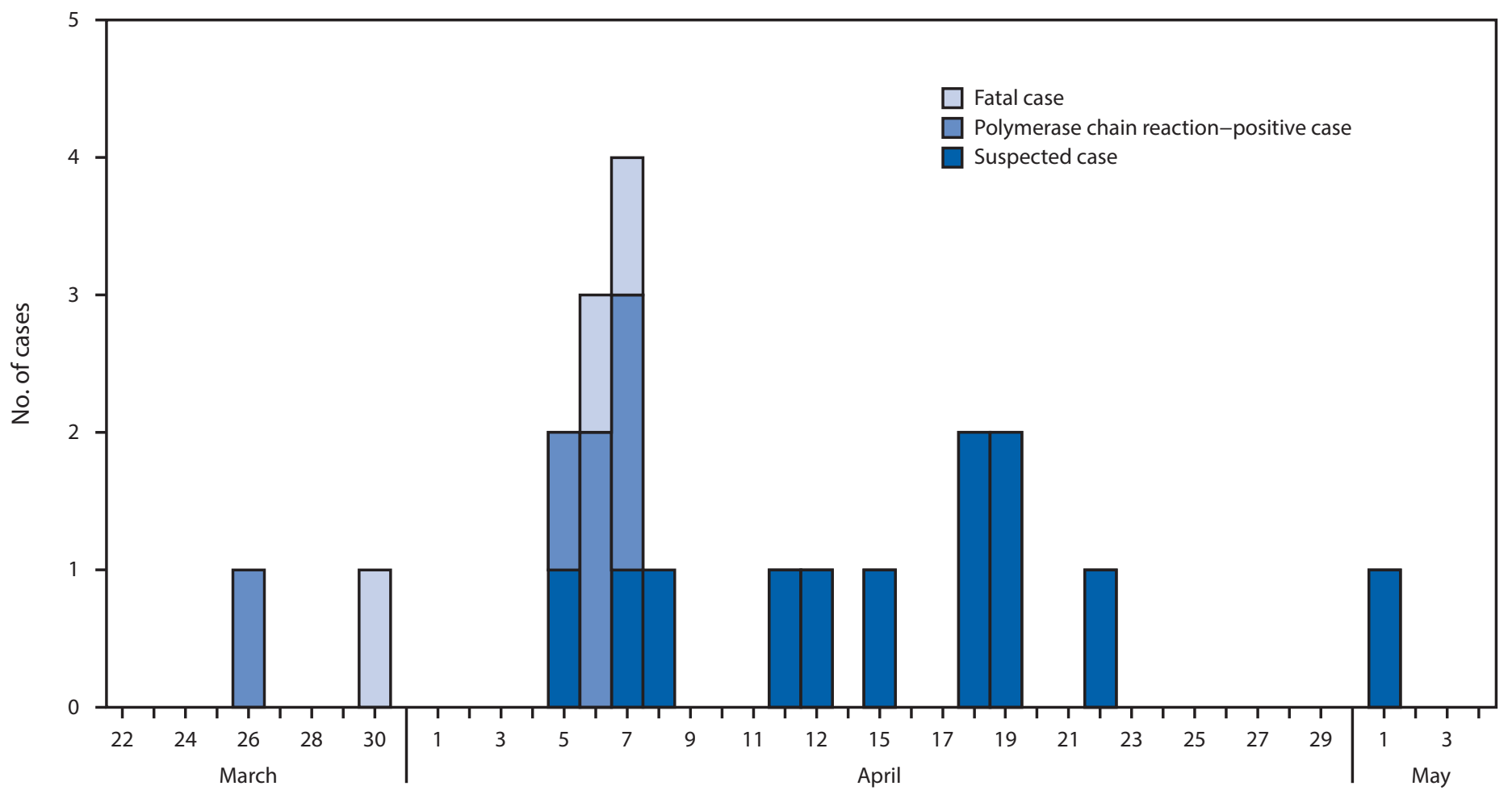

Symptom onset date

Among 18 (86\%) suspected plague patients tested for malaria during initial evaluation by RDT (SD-Bioline Malaria Ag, Standard Diagnostic Incorporation, St. Ingbert, Germany), 12 (57\%) tested positive, including all six who tested PCR-positive for plague. All of the first (according to date of symptom onset) four suspected plague patients tested positive for malaria and were treated with antimalarials (coartem and arteminisin combination therapy) on their first visit. However three of the four patients had antibiotics added to their treatment regimen on their second visits to the health center. Two of the three patients who experienced this delayed initiation of antibiotic therapy died.

Structured interviews conducted with suspected plague patients or their caretakers and families of the decedents revealed that all 21 cases slept on reed mats on the floors of their huts, which was the practice among children in the village. Although no comparison group was interviewed, village elders reported that adults generally slept on beds or mats elevated off the ground.

In response to the outbreak, indoor spraying of 1,303 (96\%) households in the two rural health center catchment areas was conducted using organophosphates (fenitrothion and pirimiphos-methyl), aimed at reducing the flea population, and recommendations were made to local leaders regarding risks for plague transmission, including that no one should sleep on the floor. Heightened surveillance for possible plague cases was initiated in this catchment area.

\section{Discussion}

The first recorded outbreak of bubonic plague in Nyimba District, Zambia resulted in 21 suspected cases and three deaths, primarily among children, during March 26-May 5, 2015. Although no cases were confirmed by culture (the gold standard), six patients tested positive for $Y$. pestis by PCR. The median age of cases in this outbreak was much lower than that in previous outbreaks in Africa $(3,4)$. One possible explanation for the younger age distribution and absence of cases among adults was the practice of children sleeping on the floor, which might have brought children into closer contact with fleas, whereas adults generally slept on beds elevated off the floor. The identification of exclusively cervical buboes was unexpected, but it is not clear that examination of inguinal or axillary areas was performed consistently. A higher frequency of cervical buboes in plague cases in children compared with adults has been reported, 
TABLE 1. Demographic and clinical characteristics of 21 suspected plague cases - Nyimba District, Zambia, March-May, 2015

\begin{tabular}{lr}
\hline Characteristic & No. (\%) \\
\hline Sex & $11(52)$ \\
Male & $10(48)$ \\
Female & \\
Age group (yrs) & $6(29)$ \\
$1-4$ & $6(29)$ \\
$5-9$ & $8(38)$ \\
$10-14$ & $1(4)$ \\
$15-20$ & \\
Clinical characteristics & $21(100)$ \\
History of fever & $14(67)$ \\
Temperature $\geq 38^{\circ} \mathrm{C}$ & $19(90)$ \\
Swollen cervical lymph nodes & $8(38)$ \\
Cough & $7(33)$ \\
Headache & $4(19)$ \\
Neck ache & $3(14)$ \\
Abdominal pain & $3(14)$ \\
Sore throat & $3(14)$ \\
Vomiting & $2(9)$ \\
Diarrhea & \\
Malaria rapid diagnostic test & $12(57)$ \\
Positive &
\end{tabular}

although the inguinal region was the most common bubo location in all age groups (3).

A large proportion (57\%) of cases, including all three fatal cases and all six PCR-positive plague cases, also tested positive for malaria. Malaria positivity might have delayed diagnosis and initiation of antibiotic treatment in some early cases in this outbreak, which might have contributed to poor outcomes. Malaria is often overdiagnosed in regions of Africa with endemic malaria, potentially delaying appropriate therapy for other bacterial illnesses (5). In areas where malaria is highly endemic, parasitemic patients can be acutely coinfected with another pathogen (G), including $Y$. pestis (7). Malaria parasitemia in Nyimba is high (21.2\%) (8), so testing of febrile patients for malaria is routine. The availability of malaria RDTs in health facilities might influence a health provider to treat an RDT-positive patient for only malaria without considering bacterial coinfections. Malaria treatment guidelines published by the Zambia Ministry of Health* and the World Health Organization ${ }^{\dagger}$ recommend antibiotics for all patients with severe disease. This recommendation could be strengthened by explicit guidance that antibiotic treatment of severely ill patients should be administered even if the patient tests positive for malaria. The antibiotic regimens that were given to the suspected cases were appropriate for the

\footnotetext{
* Government of the Republic of Zambia, Ministry of Health. Guidelines for the Diagnosis and Treatment of Malaria in Zambia. http://apps.who.int/ medicinedocs/documents/s22367en/s22367en.pdf.

$\dagger$ World Health Organization. http://apps.who.int/iris/ bitstream/10665/162441/1/9789241549127_eng.pdf.
}

\begin{abstract}
Summary
What is already known about this topic?

Plague is a bacterial disease of rodents caused by Yersinia pestis, and approximately $90 \%$ of cases are reported from Africa. Sporadic plague outbreaks have occurred in Zambia since 1917. Plague is transmitted to humans through the bite of an infected flea. Case fatality rates for untreated plague range from $66 \%-$ 93\%; prompt treatment with antimicrobials can be lifesaving.

What is added by this report?

During a plague outbreak in the Eastern Province of Zambia during March-May 2015, 21 patients with illnesses clinically compatible with plague were identified, three of whom died. Six patients tested positive for $Y$. pestis by polymerase chain reaction (PCR); 11 blood specimens and two lymph node aspirates tested for $Y$. pestis by culture were negative. Thirteen of 21 suspected plague patients also tested positive for malaria. Most plague patients were children aged $<15$ years, and children typically slept on floors in huts.

What are the implications for public health practice?

Delayed recognition of plague in an area where malaria is prevalent might have resulted in delayed treatment and poorer outcomes. PCR testing might improve detection of plague in settings with limited microbiologic capacity. Efforts should be made to increase awareness of plague and its associated risk factors among clinicians and frontline health workers to improve prevention, early case recognition, and treatment. Children with severe malaria should also be treated with antibiotics until bacterial infection is excluded.
\end{abstract}

treatment of plague and might have reduced the possibility of developing worse outcomes.

PCR supported the plague diagnosis in Nyimba, because $Y$. pestis was not isolated from blood specimens or lymph node aspirates obtained from suspected cases. Bacterial isolation is difficult in rural Zambia because of the lack of local microbiology capacity, inexperience with specimen collection and handling, and transport delays to the reference microbiology and PCR laboratory, 217 miles (350 kilometers) away. Blood specimens from the first cases were improperly collected, and at least one culture was contaminated with skin flora, suggesting breaches in sterile specimen collection technique. $Y$. pestis grows on general nutrient-rich media, but at $98.6^{\circ} \mathrm{F}\left(37^{\circ} \mathrm{C}\right)$, its growth rate is slower than that of most other bacteria; therefore, organisms that replicated faster (9) might have masked its presence. Further, it is likely that most patients received antibiotics before specimen collection, although this was not documented. Under these challenging conditions, PCR testing might be more sensitive than culture, because it does not rely on detection of viable organisms. Microbiologic confirmation has been lacking in previous plague outbreaks in Zambia (10) and elsewhere in Africa (4). However, PCR is not considered 
TABLE 2. Summary of laboratory results for suspected plague cases — Nyimba District, Zambia, March-May, 2015 (N = 21)

\begin{tabular}{|c|c|c|c|c|c|c|c|}
\hline Case no. & Age (yrs) & $\begin{array}{l}\text { WBC (x1000) on } \\
\text { admission (field test) }\end{array}$ & $\begin{array}{l}\text { Malaria RDT } \\
\text { result (field test) }\end{array}$ & $\begin{array}{c}\text { Specimens collected for } \\
\text { transport to reference } \\
\text { laboratory }\end{array}$ & $\begin{array}{c}\text { Culture result } \\
\text { (reference laboratory) }\end{array}$ & $\begin{array}{c}\text { PCR result for } \\
\text { Y. pestis (reference } \\
\text { laboratory) }\end{array}$ & Outcome \\
\hline 1 & 6 & 11.3 & Positive & Blood & Unsuitable for culture ${ }^{\dagger}$ & Positive & Alive \\
\hline 2 & 13 & -* & Positive & None & 一 $^{*}$ & - & Dead \\
\hline 3 & 11 & -* & Positive & None & 一 $^{*}$ & - $^{*}$ & Dead \\
\hline \multirow[t]{2}{*}{4} & 4 & 12.4 & Positive & Blood & Unsuitable for culture ${ }^{\dagger}$ & Positive & Alive \\
\hline & & 一 $^{*}$ & - & Lymph node aspirate & No growth & Positive & Alive \\
\hline 5 & 13 & - & Positive & None & - ${ }^{*}$ & - & Dead \\
\hline 6 & 3 & - ${ }^{*}$ & Positive & None & - $^{*}$ & - $^{*}$ & Alive \\
\hline 7 & 10 & 5.5 & Positive & Blood & No growth & Positive & Alive \\
\hline 8 & 14 & 12.5 & Positive & Lymph node aspirate & No growth & Positive & \\
\hline 9 & 3 & - & Positive & None & - & -* & Alive \\
\hline 10 & 4 & 8.1 & Positive & Blood & Unsuitable for culture ${ }^{\dagger}$ & Positive & Alive \\
\hline 11 & 5 & - $^{*}$ & - $^{*}$ & None & 一 $^{*}$ & - $^{*}$ & Alive \\
\hline 12 & 8 & 7.9 & Positive & Blood & Unsuitable for culture ${ }^{\dagger}$ & Positive & Alive \\
\hline 13 & 11 & - & Positive & Blood & No growth & - & Alive \\
\hline 14 & 10 & - ${ }^{*}$ & Negative & Blood & No growth & - ${ }^{*}$ & Alive \\
\hline 15 & 3 & - $^{*}$ & Negative & None & - $^{*}$ & - $^{*}$ & Alive \\
\hline 16 & 8 & - ${ }^{*}$ & Negative & None & - $^{*}$ & - & Alive \\
\hline 17 & 5 & - $^{*}$ & Negative & Blood & No growth & - $^{*}$ & Alive \\
\hline 18 & 10 & 一 $^{*}$ & Negative & Blood & No growth & - $^{*}$ & Alive \\
\hline 19 & 8 & 一 $^{*}$ & Negative & Blood & No growth & - ${ }^{*}$ & Alive \\
\hline 20 & 4 & -* & - $^{*}$ & None & - $^{*}$ & - ${ }^{*}$ & Alive \\
\hline \multirow[t]{2}{*}{21} & 18 & 一 $^{*}$ & -* & Blood & $\begin{array}{l}\text { Coagulase-negative } \\
\text { staphylococcus }\end{array}$ & - $^{*}$ & Alive \\
\hline & & 一 $^{*}$ & - & Lymph node aspirate & No growth & - $^{*}$ & Alive \\
\hline
\end{tabular}

Abbreviations: $\mathrm{PCR}=$ polymerase chain reaction; $\mathrm{RDT}=$ rapid diagnostic test; $\mathrm{WBC}=$ white blood cell.

* Test not done.

† Blood specimens were unsuitable for culture because they were incorrectly collected in ethylenediaminetetraacetic acid (EDTA) containers instead of blood culture bottles.

the gold standard for plague, because false-positive results can occur with specimen cross-contamination.

The findings in this report are subject to at least three limitations. First, among 111 patients identified through active case finding, medical records of 29 (26\%) were unavailable for review, which might have resulted in underascertainment of cases. Second, only 25 patients were available for examination during their acute illness, leaving investigators to rely on review of medical records to classify cases. Although follow-up interviews with patients or their parents allowed investigators to confirm symptoms, the number of actual cases might have been underestimated because of missing and incomplete data. Finally, the lack of a control group limited the ability to assess risk factors for illness compatible with plague.

Plague is probably underdetected in Zambia because of limited clinical recognition and laboratory capacity. Had the multiple subsequent cases not raised alarm, the initial cases in Nyimba would likely have been attributed to severe malaria, and plague might not have been recognized. PCR might improve detection, but will not influence clinical management given the long diagnostic delays. A point-of-care diagnostic test could improve early diagnosis and guide appropriate therapy. Efforts to increase awareness of plague among frontline health workers can improve early case recognition and treatment.
Broad spectrum antibiotic treatment to cover other pathogens is recommended for all children with severe malaria, until a bacterial infection is excluded.

\section{Acknowledgments}

Zambia Ministry of Health; Dr. Edward Schroder, CDC, Zambia; Dr. James Mwansa, University of Zambia, Lusaka, Zambia; Dr. Paul Mead, CDC, Fort Collins, Colorado.

\footnotetext{
${ }^{1}$ Zambia Field Epidemiology Training Program, Lusaka; ${ }^{2}$ CDC, Lusaka, Zambia; ${ }^{3}$ Ministry of Health, Lusaka, Zambia; ${ }^{4}$ University Teaching Hospital, Lusaka, Zambia; ${ }^{5}$ University of Zambia School of Medicine, Lusaka, Zambia; ${ }^{6} \mathrm{CDC}$, Atlanta, Georgia; ${ }^{7}$ University of Zambia School of Veterinary Medicine, ${ }^{8}$ Department of Medicine, University of Maryland School of Medicine.

Corresponding author: Nyambe Sinyange, bsinyange@gmail.com, 260-977-430-267.
}

\section{References}

1. Mwase E, Mwansa J, Musonda M. Plague outbreaks in Zambia: an overview. Zambian Journal of Medicine and Health Sciences 1999;3:50-4.

2. Ziwa MH, Matee MI, Kilonzo BS, Hang'ombe BM. Evidence of Yersinia pestis DNA in rodents in plague outbreak foci in Mbulu and Karatu Districts, northern Tanzania. Tanzan J Health Res 2013;15:152-7. http://dx.doi.org/10.4314/thrb.v15i3.1

3. Boisier P, Rahalison L, Rasolomaharo M, et al. Epidemiologic features of four successive annual outbreaks of bubonic plague in Mahajanga, Madagascar. Emerg Infect Dis 2002;8:311-6. http://dx.doi.org/10.3201/eid0803.010250

4. CDC. Bubonic and pneumonic plague-Uganda, 2006. MMWR Morb Mortal Wkly Rep 2009;58:778-81. 
5. Crump JA. Time for a comprehensive approach to the syndrome of fever in the tropics. Trans R Soc Trop Med Hyg 2014;108:61-2. http://dx.doi. org/10.1093/trstmh/trt120

6. Kibuuka A, Byakika-Kibwika P, Achan J, et al. Bacteremia among febrile Ugandan children treated with antimalarials despite a negative malaria test. Am J Trop Med Hyg 2015;93:276-80. http://dx.doi.org/10.4269/ ajtmh.14-0494

7. McClean KL. An outbreak of plague in northwestern province, Zambia. Clin Infect Dis 1995;21:650-2. http://dx.doi.org/10.1093/ clinids/21.3.650
8. Government of the Republic of Zambia, Ministry of Health. Zambia national malaria indicator survey 2015. Lusaka, Zambia: Government of the Republic of Zambia, Ministry of Health; 2015.

9. Smego RA, Frean J, Koornhof HJ. Yersiniosis I: microbiological and clinicoepidemiological aspects of plague and non-plague Yersinia infections. Eur J Clin Microbiol Infect Dis 1999;18:1-15. http://dx.doi. org/10.1007/s100960050219

10. World Health Organization. Human plague in 1997. Wkly Epidemiol Rec 1999;74:340-4. 